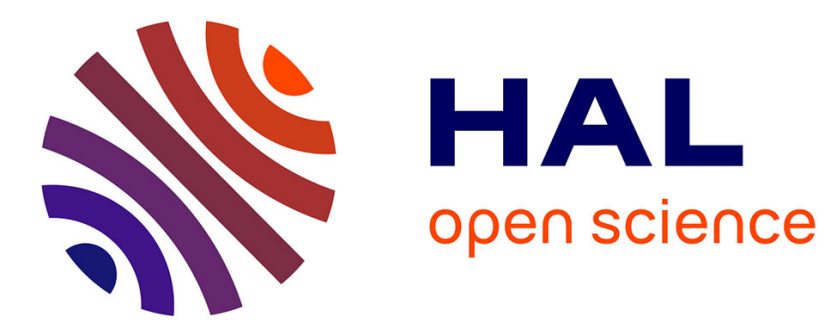

\title{
Sub-millimeter measurement of finite strains at cutting tool tip vicinity
}

Thomas Pottier, Guénaël Germain, Madalina Calamaz, Anne Morel, Dominique Coupard

\section{- To cite this version:}

Thomas Pottier, Guénaël Germain, Madalina Calamaz, Anne Morel, Dominique Coupard. Submillimeter measurement of finite strains at cutting tool tip vicinity. Experimental Mechanics, 2014, 54 (6), pp.1031-1042. 10.1007/s11340-014-9868-0 . hal-01022455v2

\section{HAL Id: hal-01022455 \\ https://hal.science/hal-01022455v2}

Submitted on 19 Apr 2018

HAL is a multi-disciplinary open access archive for the deposit and dissemination of scientific research documents, whether they are published or not. The documents may come from teaching and research institutions in France or abroad, or from public or private research centers.
L'archive ouverte pluridisciplinaire HAL, est destinée au dépôt et à la diffusion de documents scientifiques de niveau recherche, publiés ou non, émanant des établissements d'enseignement et de recherche français ou étrangers, des laboratoires publics ou privés. 


\title{
Sub-Millimeter measurement of finite strains at cutting tool tip vicinity
}

\author{
T. Pottier ${ }^{a, b *}$, G. Germain ${ }^{b}$, M. Calamaz ${ }^{c}$, A. Morel $^{b}$, D. Coupard $^{c}$
}

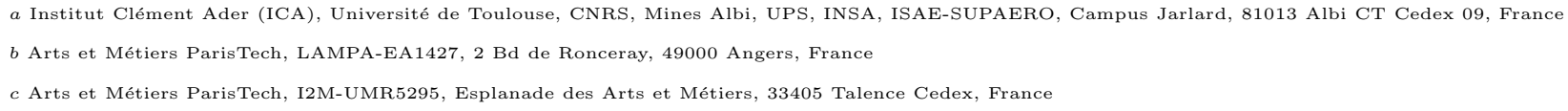

KEYWORDS

Orthogonal Cutting

Digital Image Correlation

Chip Segmentation

Adiabatic Shear Band

Machining

\begin{abstract}
The present paper details a simple and effective experimental procedure dedicated to strain measurement during orthogonal cutting operations. It relies on the use of high frame-rate camera and optical microscopy. A numerical post-procedure is also proposed in order to allow particle tracking from Digital Image Correlation (DIC). Therefore strain accumulation within finite strains framework is achieved. The significant magnitude of the calculated strains is partially due to a singular side effect that leads to local material disjunction. The strain localization in the Adiabatic Shear Band (ASB) exhibits different strain paths at various locations along this band and a non-linear evolution of the strain accumulation. A focus is made on the formation mechanisms of serrated chips obtained from Ti6Al4V titanium alloy. The side observation performed during this work allow to proposed three possible scenarios to explain this very phenomenon.
\end{abstract}

\section{Introduction}

To achieve high performance machining, it is of high interest to collect data about the chip formation mechanisms. They can help to understand and improve the integrity of the machined surface, e.g. roughness, geometrical dimensions, microstructure, residual stresses, deformation levels, as well as the wear resistance of the tool [30]. To obtain quantitative data concerning transient strain, strain rate and temperature fields in very small areas (the primary $Z_{I}$, secondary $Z_{I I}$ and tertiary $Z_{I I I}$ deformation zones), numerical simulations have been developed which enable these parameters and their evolution to be evaluated. The results only have meaning if suitable friction laws [3, 11] and material models [7, 22, 39] are used. They are currently validated merely by macroscopic data, such as cutting forces, chip morphology and tool/chip contact length. Thus, details on the behavior in the cutting zone are still lacking due to the difficulty, through experimentation, for industrial cutting parameters, to obtain local data that may link with the simulations.

Direct measurements of deformation field parameters, such as velocity of material flow and strain, have mostly been performed by quick-stop devices (QSDs). The fundamental idea is to retract the tool from the workpiece [24] or to accelerate the workpiece to separate it from the tool [6] as rapidly as possible, in order to freeze the process. [25] investigated the mechanics of chip segmentation in machining of a ferritopearlitic steel using an explosive QSD. They found that the chip segmentation process arises as a result of instabilities in the cutting process. [24] obtained clear photographs of chip roots on an AISI 1045 steel. Following the shear strain representation proposed by $[15,28]$ they predicted strain in the primary shear zone. Another way to estimate strains is to

\footnotetext{
*Tel. +33 (0)563493048
}

e-mail: thomas.pottier@mines-albi.fr

www.institut-clement-ader.org mark the material with micro grids inscribed upon the side of the workpiece by mechanical abrasion [10], chemical abrasion or photo-etching lithographic printing methods as used by [37]. The velocity and strain values are estimated from the micro grids distortion. [9] proposed a variation of the grid deformation technique, which could be used in machining, using steel specimens that contained pearlite 'stringers'. The stringers served as intrinsic internal grids whose distortion can be measured to estimate the strain. However a major drawback of QSDs is the time necessary to interrupt the cutting process. Even if improvements have been made in devices design [24], this delay time can trigger a modification of the deformation state in the cutting zone. Moreover experiments are very time-consuming since achieving a complete restitution of the formation sequence requires quick stops at various deformation stages.

An alternative to QSDs is the use of high-speed cameras. Indeed new performances in terms of acquisition frequencies, frame rate, exposure time, image resolution and signal-to-noise ratio, now allow to work close to industrial cutting speed. [37] used such a technique on a square grid marked $42 \mathrm{CrMo} 4$ steel. They managed to analyze the deformed squares at a cutting speed up to $300 \mathrm{~m} / \mathrm{min}$, deduced strains and consequently strain rates in the primary shear zone on the hypothesis of plane strain conditions. Nevertheless, at such cutting speed, the capture apparatus is only able to render 1.5 images per segment and thus only provides a glance at a more complex process. More recently, [29] proposed a method to replace the grids using flow line shape analysis. Four lines were drawn parallel to the cutting direction on the side of the workpiece using a mechanical scratching method. The velocity, strain rates and strain distributions were calculated from the mathematical expression 
of the streamline functions. Orthogonal cutting tests were conducted up to $1020 \mathrm{~m} / \mathrm{min}$. But a high feed of $0.84 \mathrm{~mm}$ had to be selected to guarantee a sufficient spatial resolution and avoid an underestimation of strain. The use of Digital Image Correlation (DIC) has also been reported to analyze the cutting zone. [21] have developed a complex dedicated device composed of four non-intensified digital cameras set in dual frame mode to perform a 4 images acquisition at $1 \mathrm{MHz}$.

In the present paper, DIC technique has also been chosen. It is performed from the use of a single high frame rate imager and optical microscopy. Such experimental apparatus allows to monitor strain at several instants during the chip formation process. The following therefore presents first the experimental procedure. Details are given on cutting configuration and observation device. Then the DIC post-processing is described. Its accuracy is discussed. A following section is dedicated to the numerical estimation of strain from displacement fields. Finally the obtained results are presented and discussed. Mechanisms of chip formation are then proposed based on these experimental data.

\section{Experimental setup}

\section{Material and cutting characteristics}

The cutting configuration is chosen to be orthogonal. Cutting tests are performed on a planer machine GSP 2108 R.20 which allows varying the cutting velocity between 6 and 60 $\mathrm{m} / \mathrm{min}$. Uncoated carbide inserts with a tool holder manufactured for the planer machine (rake angle : $0^{\circ}$ ) are used. The tool is fixed and the cutting speed is applied to the machine table on which the workpiece is set up, along a direction perpendicular to the cutting edge (orthogonal cutting operation). The workpiece moves perpendicular to the observation axis.

The width of cut $(w=3 \mathrm{~mm})$ is given by the dimensions of the Ti6Al4V workpiece $(100 \times 60 \times 3)$. A cutting depth of $f=0.25 \mathrm{~mm}$ is selected. This choice results from a balance between the need of large shear zone (lower magnification required) and the need to limit the out-of-plane effects (optical depth of field). The instruction of cutting speed is set to $V_{c}=6 \mathrm{~m} / \mathrm{min}$. These cutting parameters are expected to generate serrated chips (see Fig.1-c) on a material well known for segmenting even at low cutting speeds [25, 38].

\section{Optics and field measurements}

One of the main improvement of the present study is the use of high magnification optics allowing the capture of submillimeter zones of interest. For instance, in [19] the use of a $3 \mathrm{X}$ optical microscope allows a pixel size of $8.2 \mu \mathrm{m}$ at 250 $f p s$, in [20] the use of a high definition camera and a similar magnification allows a pixel size of $1.4 \mu \mathrm{m}$ at $2000 \mathrm{fps}$. The work of [37] proposes the use of a $12 \mathrm{X}$ video-microscope for a pixel size of $4.9 \mu \mathrm{m}$ at $25000 \mathrm{fps}$. Finally, [21] proposes to use 4 standard CCD sensors triggered successively in order to obtain 4 images with an inter-frame time of $0.001 \mathrm{~ms}$ (equivalent to $1 M f p s$ ) at very high magnification (pixel size: $0.27 \mu \mathrm{m})$. However, monitoring the chip formation process require a complete sequence of images. Therefore, the proposed capture process relies on a Photron Fastcam APX-RS camera.
The spatial resolution is a critical parameter in investigating large strain gradients, especially when localization phenomena are involved. The use of high speed camera limits quite drastically the number of measured pixels. Subsequently, the link between frame rate and frame resolution is responsible for limiting the maximum cutting speed of an observation. The choice of the capture parameters is governed by the will to obtain several images of one segment formation. In order to keep a sufficient spatial resolution, the acquisition is set at $f=18000 \mathrm{fps}$ with an exposure time of $6.6 \mu \mathrm{s}$. This setting ensures a resolution of $384 \times 352$ pixels.

A tunable optical video-microscope is used and its magnification $\left(M_{t}\right)$ is set to $35 \mathrm{X}$. The field of view is then $650 \times 600 \mu \mathrm{m}$, with a pixel size of $1.65 \mu \mathrm{m}$. With such settings, a cutting speed of $V_{c}=6 \mathrm{~m} / \mathrm{min}$ provides about 45 images of one segment formation (assuming that the width of a segment can roughly be approximated by $f=0.25 \mathrm{~mm}$ ). One should also notice that such an optical apparatus is responsible for optical aberrations of unknown nature. Under this condition, one should be careful in the use of DIC when estimating the correlation between a reference (undeformed) image and a deformed one that has undergo serious displacement within the aberration field. Accordingly, iterative correlation should be preferred (see section).

Lighting the scene is a non-trivial issue and deserve to be discussed further here. The presented images are captured using a $200 \mathrm{~W}$ halogen light source and an optic-fiber light guide. Set at full power this device is not sufficient to capture images with an exposition time of $6.6 \mu s$. Actually the beam splitter embedded within the video-microscope is responsible for a significant loss of the incoming light flux (indeed the half of it) Therefore the light guide was positioned outside of the microscope tube, about $5 \mathrm{~mm}$ away from the observed scene. The approximated angle of incidence is $30^{\circ}$. Nevertheless, lighting remains one of the main constraint in high-speed imaging especially under microscopic conditions where over-exposure and/or under-exposure may become significant issues.

\section{Digital Image Correlation}

\section{Calculation parameters}

The whole captured sequence is made of 2568 frames representing the formation of 52 chip segments. However, only one segment formation has been selected for post-processing purpose. Hence, 45 images are investigated. Each image is postprocessed through Digital Image Correlation (DIC) in order to retrieve the incremental displacement field. For this purpose, each image is split in square elements that create a virtual grid upon the sample surface (denoted $\mathcal{M}_{0}=\left\{x_{0} ; y_{0}\right\}$ ). The resolution of this grid (the gauge length) is set to $10 \times 10$ pixels, corresponding to $17 \times 17 \mu m^{2}$.The correlation process consists in looking for the most probable deformed pattern in the neighborhood of each node of this grid in terms of grey level. The displacement fields of each element are then assessed by the means of a bi-linear interpolation. Finally, the displacement at image $n$ is assessed by correlating the $n^{\text {th }}$ image with the previous image $(n-1)^{t h}$. A zero-mean formulation of the correlation parameter is used in order to prevent from error due to non-constant lighting. The correlation parameter used in the present study are summarized in Table 1. Calculations are performed using $7 D$ software [43]. 


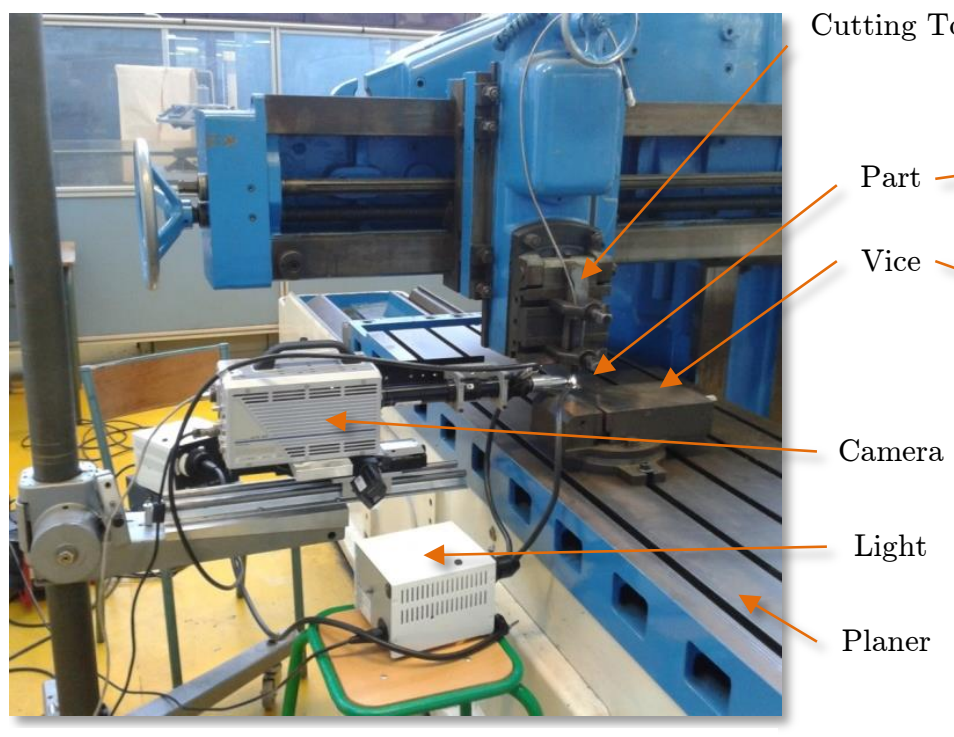

a)

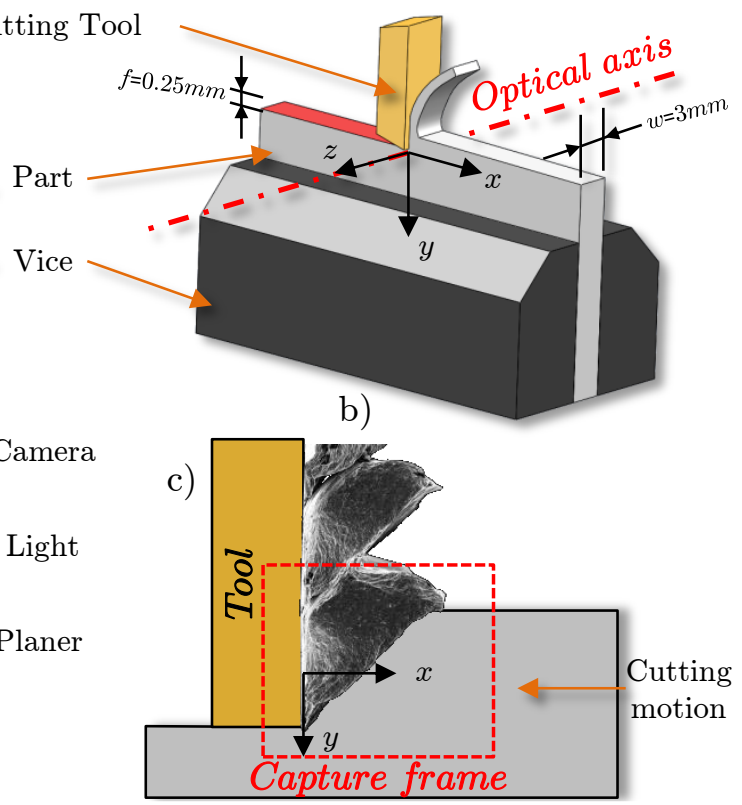

Figure 1: a) - Experimental apparatus [18]. b) - Geometry of the cutting and coordinates system. c) - Side view of the scene showing the camera capture frame and the serrated chip (SEM view overlaid).

Table 1: Main parameters of the Digital Image Correlation

\begin{tabular}{cccc}
\hline $\begin{array}{c}\text { Grid } \\
\text { size }\end{array}$ & $\begin{array}{c}\text { pattern } \\
\text { size }\end{array}$ & $\begin{array}{c}\text { grey level } \\
\text { interpolation }\end{array}$ & $\begin{array}{c}\text { displacement fields } \\
\text { interpolation }\end{array}$ \\
\hline $10 \times 10$ & $10 \times 10$ & bicubic & bilinear \\
\hline
\end{tabular}

The choice of incremental correlation relies on several considerations. Firstly, as mentioned above, the optical aberration leads to a significant error when large displacements are involved ([36]) and comparing image $n$ and $n-1$ instead of $n$ and 1 decrease such displacements. Secondly, in presence of very significant strains, the likeness of image no. 1 and $n$ is poor and the correlation process cannot return information over the whole correlation grid $\mathcal{M}_{0}$. In other words, the material fed in the right hand side of image $n$ has no counter part in image 1 for it is still out of the frame at that time.

\section{Displacement uncertainties}

Estimating the measurement uncertainties of speckle-related DIC techniques, is known to be a complex task. Lots of studies have been addressed in this field and the point of the present paper is not to discuss any further in this matter. However, the poor quality of the obtained images (low resolution, optical aberration, camera measurement noise, unpainted surface) give raise to the question of the accuracy/noise of the DIC obtained displacement fields. Among the several parameters impacting the DIC accuracy, speckle quality and pattern size are often addressed as the most critical ones $[4,33]$.

The benchmark speckles generated numerically in [4] offer a fast way to qualitatively investigate a given speckle. Indeed the estimation of the autocorrelation of the texture allows a comparison with the 3 proposed speckle. Fig.2-a) show the texture and the autocorrelation surface of a $10 \times 10$ pattern. Fig.2-b) depicts a comparison of the speckle under investigation with those proposed in [4]. Hence, it can be seen that the obtained texture exhibits a sufficient quality to ensure a proper DIC (though between standard and coarse).

Another approach in evaluating speckle quality is the use of rigid body motion [42]. A non-deformed zone of the image is investigated over 5 successive images and the displacement norm is monitored. The average displacement in the zone is then subtracted. Subsequently, the residual stands for the noise generated by speckle imperfection. Alike any random variable, it can be characterized by its standard deviation. Fig.2-c) shows that for the chosen pattern size $(10 \times 10)$ the displacement uncertainties can be assumed to be about 0.0181 pixels $\approx 0.031 \mu \mathrm{m}$ (say a signal-to-noise ratio of 165 in the non-deformed zone).

Finally, the standard deviation of the measured displacement can also be estimated through the Mean Intensity Gradient (MIG) of the speckle pattern([32]). It is shown that under some assumptions, the random error is given by:

$$
\operatorname{std}(u) \approx \frac{\sqrt{2} \sigma}{n \times \delta_{f}}
$$

where $\sigma$ is the standard deviation of the measurement noise, $N$ the subset size and $\delta_{f}$ the MIG. In the present study the MIG equals $\delta_{f}=9.22$ and the noise $(\sigma=1.025$ pixel $)$ is evaluated from the substraction of two consecutive images captured prior to any motion of the sample. Hence the standard deviation of the measured displacment is approximated by: $\operatorname{std}(u) \approx 0.0157$ pixels $\approx 0.027 \mu m$ which is consistent with the rigid motion approach.

\section{Out-of-plane motion}

Unlike the work presented in [19], the cutting geometry does not impose constraint on the observed side of the chip. It 

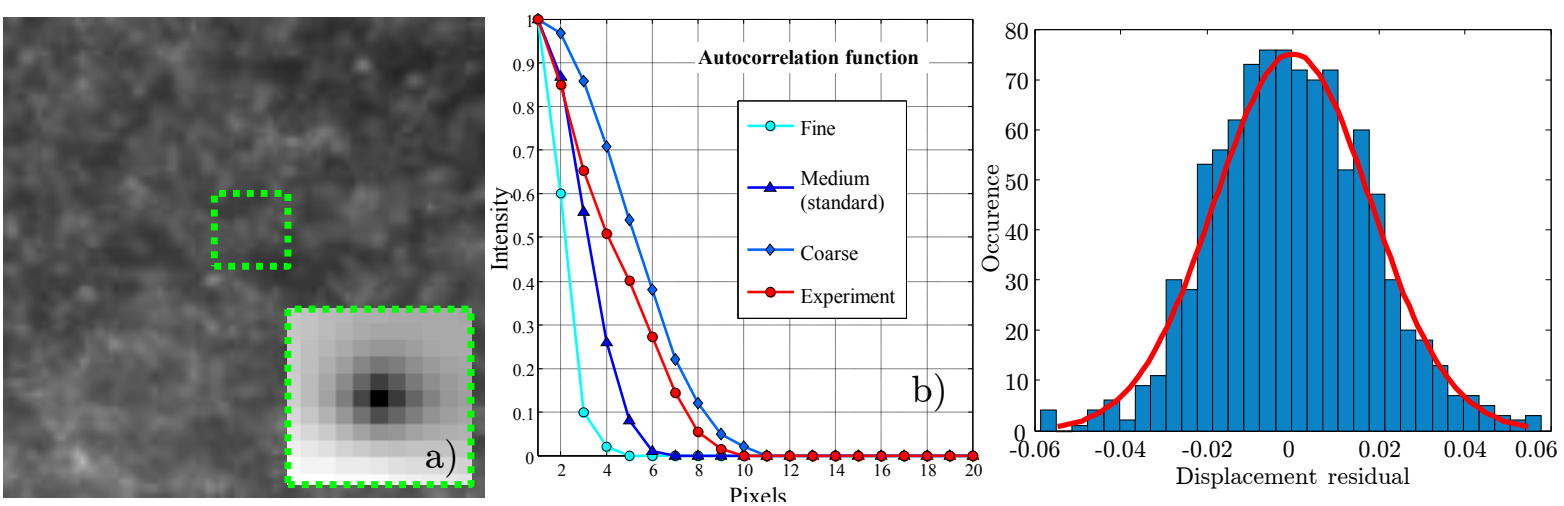

Figure 2: a) Image detail window $100 \times 100$. and autocorrelation zoom $10 \times 10$. b) Associated centered and normalized autocorrelation function radius at half height [4]. c) Distribution of normalized displacement vector norm.

means that the chip is free to swell along the optical axis of the microscope and therefore impose accounting for the out-of-plane deformation in the sheared section. The magnitude of the out-of-plane deflection is estimated from either SEM-MEX technique (stereoscopic 3D reconstruction Fig.3a) and chromatic confocal surface metrology (Altimet 500). The approximated magnitude of the out-of-plane deflection is $\Delta z \approx \pm 35 \mu \mathrm{m}$ for both measurements. However, one should notice that this measurement is performed post-mortem and does not account for the elastic deformation of the chip which is then neglected.

Several studies have been addressed on the estimation of DIC error due to out-of-plan motion and theoretical models enable the assessment of the errors raised from such motion. The work of [41] proposes both a single lens model and a telecentric model to account for the virtual magnification of objects moving along the optical axis. The nature of the objective in used in this study (infitity corrected planapochromatic) disable the used of any of these models. Hence, the error estimation has been performed from experimental means. A planar speckled plate was moved along the optical axis and a pictured was captured every $10 \mu \mathrm{m}$ (within a range of $-40 \mu \mathrm{m}$ to $+40 \mu \mathrm{m}$ from the focus plane). DIC was then used to estimate the virtual strains due to out-of-plane motion. Fig. 3 shows that the error in terms of strains is maximized by $\varepsilon_{\max } \approx 0.0055$. Since the swelling of the chip is not homogeneous over the chip segment, some regions are more affected by this error than others. However, the small magnitude of such error compared to the estimated strains (see section ) leads to consider it as negligible in the presented study case. One should notice that this error source can be minimized by the use of telecentric optics as disscussed in [41] and [34].

\section{Strain Calculation}

\section{Finite strain Framework}

The main difficulty in estimating displacement and strain fields relies on the use of incremental correlation which disable the tracking of a given material point. For every image, the increment of displacement is given at the undeformed/reference location as

$$
\left\{\begin{array}{l}
\Delta U_{x}\left(x_{0}, y_{0}, k\right) \\
\Delta U_{y}\left(x_{0}, y_{0}, k\right)
\end{array}\right.
$$

where $\left(x_{0}, y_{0}\right)$ are the grid coordinates (identical for every image pair) and $k$ is the image number. This only information does not enable the estimation strains (whether in initial or final configuration) since material tracking can not be achieved, cumulating strain and different location is just not right. The evaluation of the deformed coordinates $\left\{x_{k} ; y_{k}\right\}$ at every step/images of the deformation process is performed through triangular bi-linear interpolation.

$$
\left\{\begin{array}{l}
x_{k}=x_{k-1}+\Delta U_{x}\left(x_{k-1}, y_{k-1}, k\right) \\
y_{k}=y_{k-1}+\Delta U_{y}\left(x_{k-1}, y_{k-1}, k\right)
\end{array}\right.
$$

with

$$
\left\{\begin{array}{l}
\Delta U_{x}\left(x_{k-1}, y_{k-1}, k\right)=\sum_{i=1}^{3} \phi_{i}\left(x_{k-1}, y_{k-1}\right) \times \Delta U_{x}\left(x_{0}, y_{0}, k\right) \\
\Delta U_{y}\left(x_{k-1}, y_{k-1}, k\right)=\sum_{i=1}^{3} \phi_{i}\left(x_{k-1}, y_{k-1}\right) \times \Delta U_{y}\left(x_{0}, y_{0}, k\right)
\end{array}\right.
$$

where the $\phi_{i}$ are the classical triangular shape functions. The knowledge of displacement increments offers a straight forward access to the velocity fields $V_{x}$ and $V_{y}$. Assuming a constant capture rates of the images, it therefore comes $V_{x}=\Delta U_{x}$ and $V_{y}=\Delta U_{y}$. Then the components of the strain rate tensor are:

$$
\left\{\begin{array}{l}
\mathbb{D}_{x x}\left(x_{k}, y_{k}, k\right)=\frac{\partial V_{x}\left(x_{k}, y_{k}, k\right)}{\partial x_{k}} \\
\mathbb{D}_{y y}\left(x_{k}, y_{k}, k\right)=\frac{\partial V_{y}\left(x_{k}, y_{k}, k\right)}{\partial x_{k}} \\
\mathbb{D}_{x y}\left(x_{k}, y_{k}, k\right)=\frac{1}{2}\left(\frac{\partial V_{y}\left(x_{k}, y_{k}, k\right)}{\partial x_{k}}+\frac{\partial V_{x}\left(x_{k}, y_{k}, k\right)}{\partial y_{k}}\right)
\end{array}\right.
$$

The cumulated displacement at material point location is then used to calculate the updated Lagrangian strain tensor as:

$$
\mathbb{E}\left(x_{k}, y_{k}, k\right)=\mathbb{D}\left(x_{k}, y_{k}, k\right)+\sum_{m=1}^{k} \sum_{i=1}^{3} \phi_{i}\left(x_{k}, y_{k}\right) \times \mathbb{D}\left(x_{m}, y_{m}, m\right)
$$

where $1<k<N_{f}$ and $N_{f}$ is the number of frame in the sequence. $\mathbb{E}$ is then the cumulated strain with respect to the 


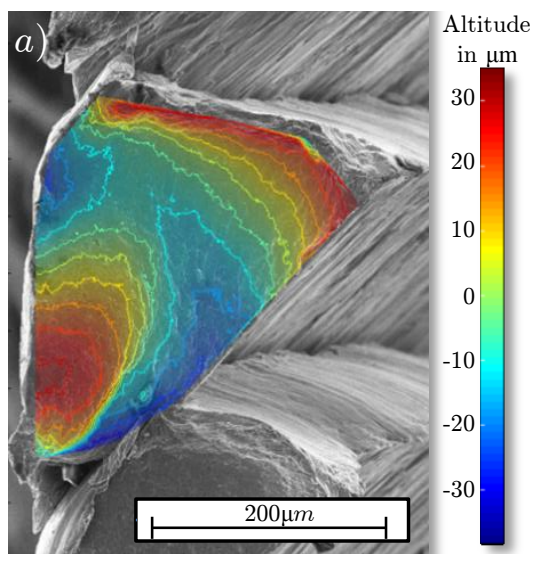

b)

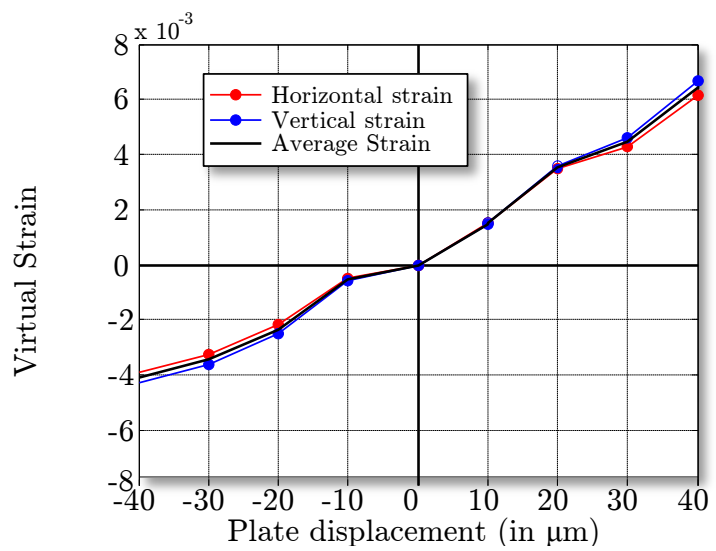

Figure 3: a) SEM image of the chip side, overlaid with the measured altitude map. b) Error estimation on strains due to out-of-plane motion.

initial configuration but expressed over the current deformed grid $\left\{x_{k} ; y_{k}\right\}$ (Updated Lagrangian Configuration). Such a formulation allows a straight forward comparison with standard Finite-Element commercial codes and is therefore well suited for model validation and/or identification.

\section{Modal derivation}

The major issue related to the implementation of Eq.(6) comes from the noisy nature of displacements obtained by DIC approaches which lead to significant signal-to-noise ratio (SNR) when derived. Such numerical instabilities prevent from direct derivation of displacements when these latter exhibit high SNR, e.g.: natural speckle, small spatial resolution, inconsistent lighting, strain localization.. All of these problems arises with the combined use of high-speed photography and high magnification. Hence, filtering method should be considered and implemented.

In the present study, a modal projection method is used [35]. This method consist in projecting the displacement fields within a modal basis and build the strain fields from a weighted sum of modal space-derivative. The displacement increment is written as:

$$
V_{\mathbf{x}}\left(x_{k}, y_{k}, k\right)=\sum_{p=1}^{N} \lambda_{p}(k) Q_{p}\left(x_{k}, y_{k}\right)
$$

where $N$ is the truncation order (the number of modes used for reconstruction) here set to $350, \lambda_{p}$ the modal coordinates and $Q_{p}$ the modal vectors. These latter are here evaluated from dynamics vibration theories. Indeed, prior to any calculation, the basis $\mathfrak{B}=\left\{Q_{1}, Q_{2} \ldots Q_{N}\right\}$ is calculated by solving the classical dynamics problem:

$$
\left(M^{-1} K-\frac{1}{\omega_{i}} \mathbb{I}\right) Q_{i}=0,
$$

where $M$ is the mass matrix, $K$ the stiffness matrix and $\omega_{i}$ the modal pulsation. The problem is solved using Finite Element discretization over a mesh build using one node per pixel of the captured image.

Once the velocity fields are projected within $\mathfrak{B}$ the linearity of the derivative operator states that:

$$
\mathbb{D}_{i j}\left(x_{k}, y_{k}, k\right)=\frac{\partial V_{i}}{\partial x_{j}}=\sum_{p=1}^{N} \lambda_{p}(k) \frac{\partial Q_{p}\left(x_{k}, y_{k}\right)}{\partial x_{j}}
$$

This approach, though uncommon in strain derivation applications, has proved its worth in various noise related problems $[12,45,27]$.

\section{Results and discussion}

\section{Cumulated strain in a segment}

Fig. 4 shows the shape of the displacement and principal strain fields during chip segment formation. Adiabatic shear bands (ASB) are clearly highlighted by significant strain magnitudes (up to 3 ) at these locations. In addition, it can be noticed that the inner part of the segment does not significantly deforms (columns $c$ ) and $d$ ) image no.45).

Moreover, the actual cutting speed can be evaluated from the actual displacements increments measured in an undeformed zone of the image. For this purpose the displacement norm within the lower right side of the image (see Fig.4-a) is averaged and gives $V_{c} \approx 5.63 \mathrm{~m} / \mathrm{min}$ which is slightly lower than the instruction $\left(V_{c}=6 \mathrm{~m} / \mathrm{min}\right)$.

As mentionned in the above, the measured strains are significant. However, a close monitoring of the image sequence shows the outbreak of a localized black line (Fig.5-a). In order to determine the nature of this phenomenon, complementary SEM investigations have been carried out. As shown in Fig.5$\mathrm{b}$, a material disjunction (or crack) appears on the free side of the chip.

The fact that, at some point of the deformation process, the material splits in segments leads to reconsider the strains magnitude assessment. The DIC process such as presented in this paper keeps cumulating strains even though material undergoes segmentation. Hence, the final strain magnitude within ASB is not relevant as such from a continuum mechanics standpoint. The values (up to 3) as depicted in Fig.4-cd) are indeed greatly overestimated. It is then required to monitor the outbreak of this material disjunction.

For this purpose, the study of the strain rates within the ASB as depicted in Fig.6 provides a straight forward way to monitor the crack propagation. From the curves presented in Fig.6-b) it is noticeable that a segment is formed from three successive stages.

Stage 1 - The strains are concentrated at the tooltip vicinity. The inner part of the chip slowly deforms (Fig.4-d). 

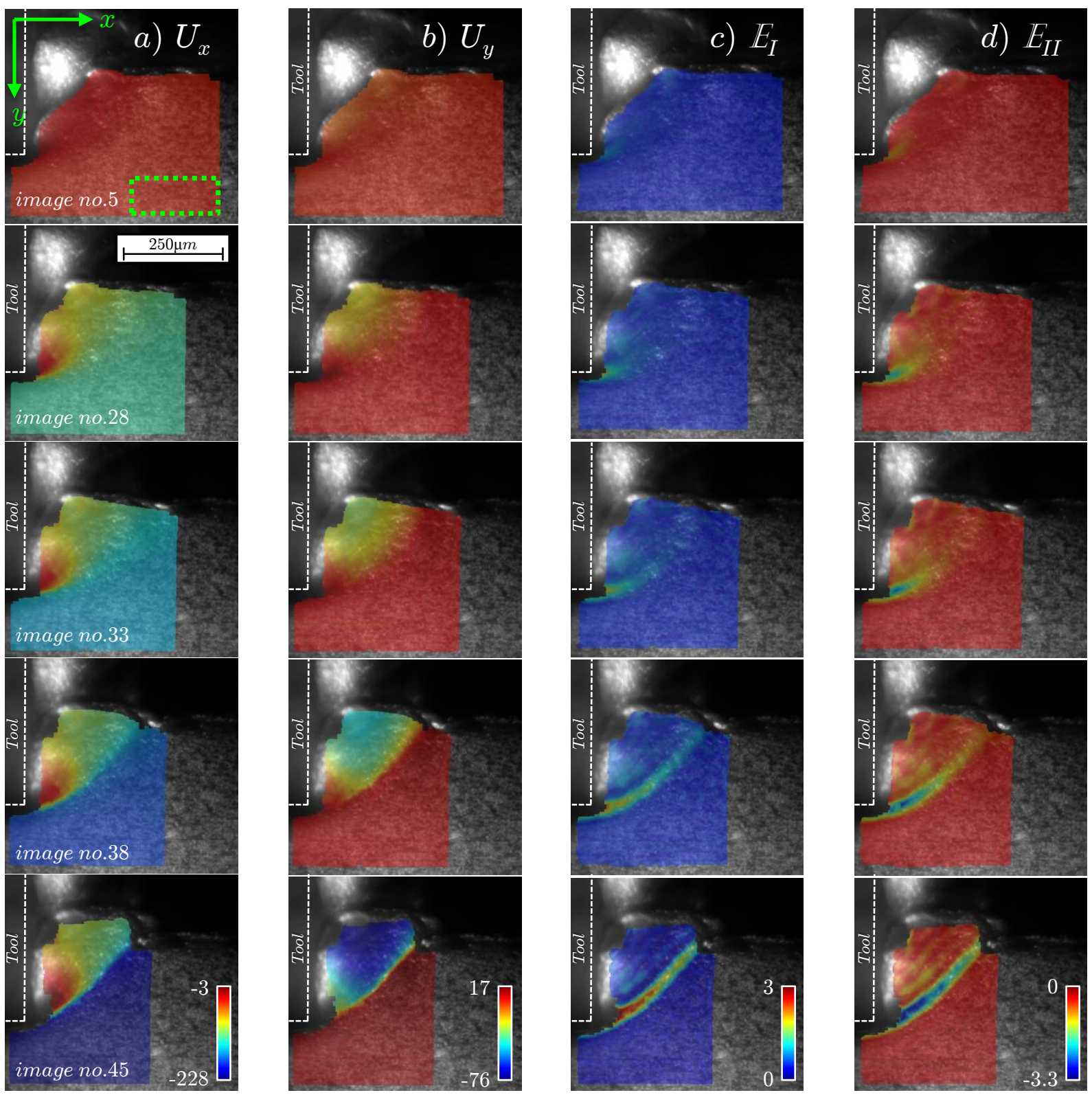

Figure 4: Displacements and principal strain fields at five time steps. The total elapsed time is 2.2 ms corresponding to the formation of one segment.a) Horizontal displacement (in $\mu \mathrm{m}$ ). b) Vertical displacement (in $\mu$ m). c) Major strain. d) Minor strain. The green rectangle represent the zone where displacements are averaged for the cutting speed estimation.
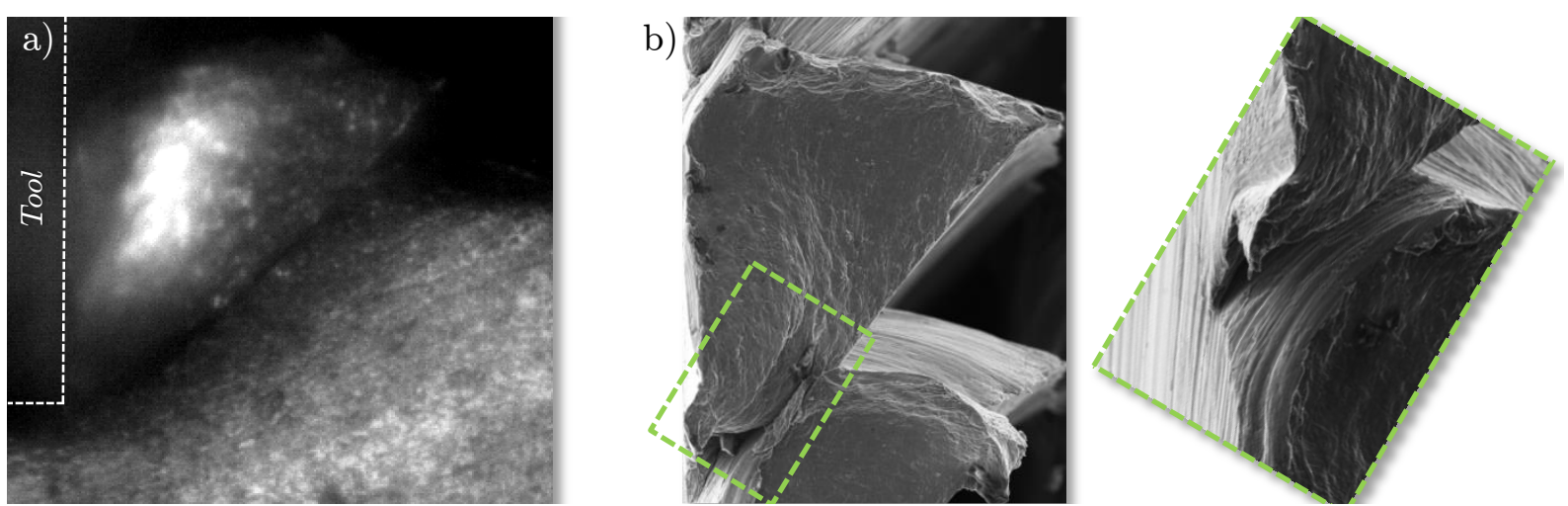

Figure 5: a) Image captured during segment formation (contrast modified to enhance black line visibility). b) SEM view of the chip side and detail view of the crack. 
During this stage an out-of-plane swelling of the segment is clearly visible. It is fully formed around image no.30.

Stage 2 - The strain rapidly cumulates along the ASB from bottom to top. The strain rates reach significant magnitudes (up to $4.10^{3} s^{-1}$ ) and lead to a material failure (this latter point is specifically discussed in section )

Stage 3 - During the last images, strain rates stabilize, the segment is fully formed and slips on both the cutting face and the next segment (to be formed). Indeed, since material failure occurs the segment is animated with a rigid body motion. At this point, cumulating strains is no longer valid from a continuum mechanics standpoint.

Fig.6-b also shows that the times spent for stage 2 goes shorter when moving from point 1 to point 3 . In other words, the material failure accelerates from the tooltip to the free surface. Considering the decreasing of the resisting cross-section, this results is indeed expectable.

Fig. 7 shows the last measured state of strain before material failure (at the end of stage 2) for 19 points along the ASB. It can be seen that the deformation process that leads to failure slightly differs from one end of the ASB to the other. Different strain paths are observed and can be sorted in three categories:

Zone $Z_{I A}$ : Nearby the tooltip, the ASB deforms mainly in compression with significant magnitudes. This process is slow and start very early in the sequence (Fig.6-b, point $P 1$ and Fig.7-b).

Zone $Z_{I B}$ : Moving away from the tool tip, the ASB deforms in compression/shear but with lower magnitudes. The strain rates curves in Fig.7-c show an acceleration of the phenomenon: the material is less deformed at failure in this zone but reaches it faster.

Zone $Z_{I C}$ : Finally, around the free surface, the state of strain turns into pure shear. At this time of the sequence the cutting speed reaches a cycle maximum. It is noticeable that the resisting cross section tends toward its minimum at the same time. It seems plausible that the two observations are related, however only a rigorous measurement of the cutting force may validate such hypothesis.

The study of strain rates enables to follow the crack tip when propagating along the ASB. This criterion, though unautomated yet, corroborates the visual observations of the images sequence. It allows to limit the strain accumulation and evaluate strains at failure. These latter appears to be heterogeneous and lower than expected by numerical calculation $[40,8]$. These observations points the need of a better understanding of the decohesion phenomenon and the segmentation process.

\section{Chip segmentation process}

The development of numerical models of machining operations has become significant in the last decade and the modeling of the segmentation phenomena is a key issue in the validation of material models (either for constitutive models and for damage evolution laws) [7]. A close monitoring of the image sequence reveals that the crack grows from the tooltip to the top free surface over 17 images. This film shot on the side surface, clearly shows that the disjunction goes from end to end. It can be seen in Fig.8 that the material is disconnected on the side from $\delta=10 \mu \mathrm{m}$ while that disconnection appear at the center at approximately $70 \mu \mathrm{m}$. Say, a small part of the cross-section (in the triaxial pressure zone) is still attached to the next segment. It results in the formation of a serrated chip (and not a fully segmented one). As highlighted by [26], the presence of a high hydrostatic stress zone at the chip center prevents form crack formation at the tooltip vicinity. As a matter of fact, such zone cannot exist near the free surface of the chip which is consistent with the end-to-end crack observed on the side (Fig.5-a).

The formation process of this disjunction remains largely undocumented in the literature at least from an experimental standpoint and no mention of side effects is made. However, the observations performed during this work show significant differences between the side and core disjunction processes. From the knowledge of the 3D nature of the chip formation process and other published observations, one may think of three possible scenarios to model the crack evolution:

The first scenario is depicted in Fig.9-a). The crack initiate at the tooltip (on the side), propagates along the primary shear band, generates a step on the top surface, and keeps propagating backward (through-thickness) to the triaxial pressure zone. This hypothesis is backed by chip post-mortem micrography and QSP observations of polished chips and is widely acknowledge in the scientific community $[17,44,31]$. Regretfully, these observations are made at one given time of the formation process and cannot provide evolutionary information. Moreover, the material removal due to polishing disable the observation of side effects.

The second scenario is depicted in Fig.9-b). The crack initiates at the tooltip (on the side) and while propagating along the primary shear band it turns trough-thickness when the hydrostatic pressure becomes low enough. Then, it reaches the face-side corner first, then the center of the face. This scenario is consistent with the $2 \mathrm{D}$ numerical observations performed by [23]. Authors have used the energy-based ductile fracture model proposed by [13] and performed chip micrographic observations. However, the numerical model is built under a $2 \mathrm{D}$ assumption and does not provide information on the side effects.

The third scenario, depicted in Fig.9-c), is actually a variation of the second scenario. Instead of a side initiation, the crack initiates all along the borders of the triaxial zone. One may assume that the high compressive strains at these locations make the triaxial zone behave as a rigid body that slips on the cutting face. The crack is then initiated simultaneously on the side and through-thickness. This scenario, though not as well-established as the others, may explain the existence of the material voids visible in various chip micrographic observations $[14,5]$.

Discussion among those three possibilities is a complex experimental (and numerical) task and the purpose of this work is not validate (or discard) these hypothesis. However, various observations can offer leads on that matter. For instance, the constant strain rate observed right after the completion of the side crack observed in Fig. 6 is not really compatible with the first and second scenarios (where the segment is still 

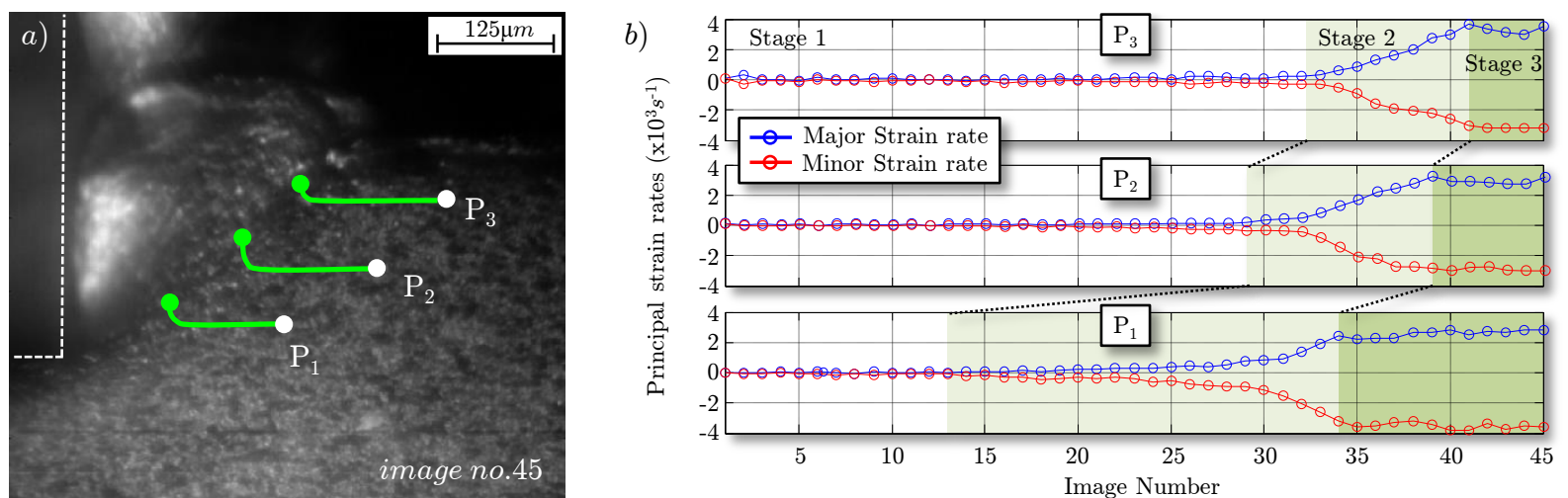

Figure 6: a) Initial and final position of the three investigated points $\left(P_{1}, P_{2}\right.$ and $\left.P_{3}\right)$. b) Principal strain rates evolution over the 45 images of the film (at $P_{1}, P_{2}$ and $\left.P_{3}\right)$.
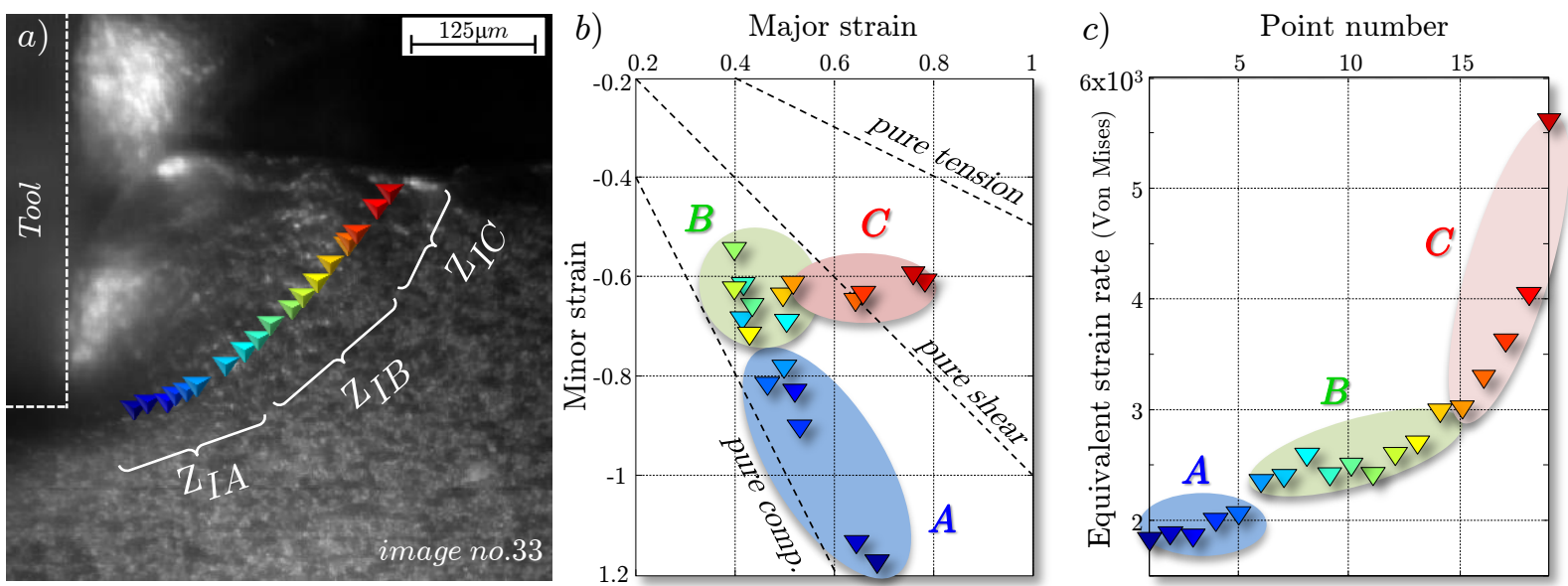

Figure 7: a) The three sub-zones of the ASB and the 19 selected points. b) State of strain at failure. c) strain rates at failure for the selected points.
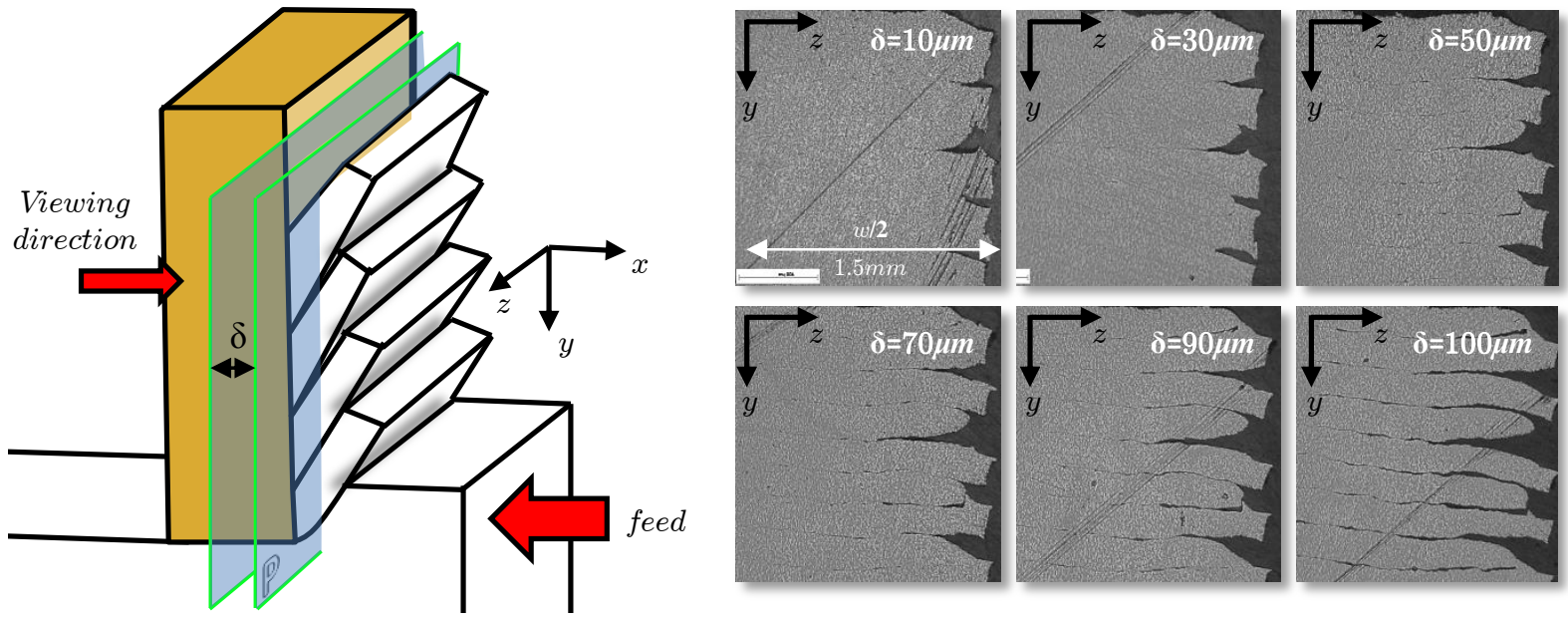

Figure 8: Successive cuts of the chip through thickness showing an open crack at the left end but connected material within the hydrostatic stress zone. 


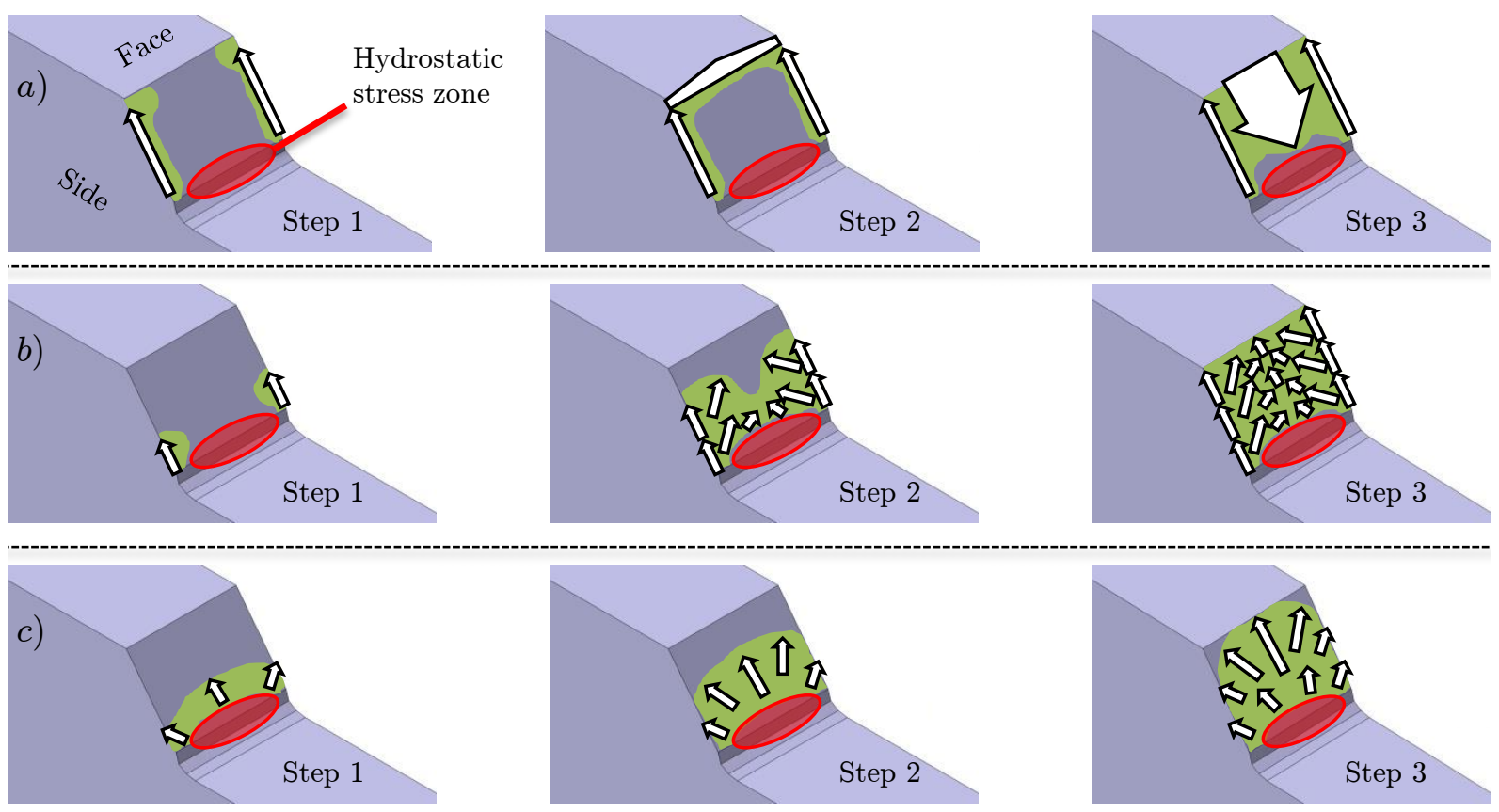

Figure 9: a) First scenario : face-to-core propagation of the crack (with side initiation). b) Second scenario : side-to-core propagation of the crack (with side initiation). c) core/side-to-face propagation of the crack with simultaneous initiation.

not fully formed when the side crack runs from end to end). The first scenario does not explain the existence of material voids along the ASB. QSDs could be a good way to investigate the crack geometry but also existence and location of material voids and side effects, upon condition that the instant of the tool separation is precisely known (high frame rate imaging can be used on that purpose). In addition, the use of in-process observations of the top face may help differentiate the 3 scenarios (outbreak of the crack on the top). Another lead would be a cutting force monitoring. It would enable to link strain rates and the evolution of the resisting cross-section. Finally, the effect of temperature is also to be investigated.

\section{Conclusion}

This paper discusses the difficulties using built-in strain measurement in machining conditions. It proposes a submillimeter procedure for image acquisition and a suitable numerical post-processing that enables strain evaluation during the segmentation process. Results show a significant strain localization within the ASB and heterogeneity of the deformation behavior along this band.

This work provides experimental evidences of a significant side effect and highlights the 3D nature of the chip (even in orthogonal cutting conditions). It therefore challenges the classical plane strain hypothesis widely acknowledged in literature. Indeed, chips are often wider than thick and this hypothesis holds as long as the averaged behavior is investigated. However, considering a chip formation process with crack initiation on the sides (where the plane stress hypothesis seems more suited) would clearly require the use of $3 \mathrm{D}$ finite element formulation.

In addition, the presented procedure offers a way to quantify the strains within the adiabatic shear band under the assumption of material continuity. However, as seen in the above, this hypothesis does not hold when serrated chip are produced. Hence, one understands that detecting the presence (or absence) of material disjunction is a serious issue and can lead to significant over-estimation of the strains. The development of a reliable criterion for crack detection (based on the orientation of the velocity gradient vector) is under construction and will be presented in future publication.

Two main drawbacks can be singled out of the presented experiments. The first one is the lack of in-process information on the magnitude and the shape of the out-of-plain deformation of the chip. It is a direct consequence of the chosen experimental procedure. Indeed, the topological study of this phenomenon is an experimental challenge of its own and constitutes a perceptive of this work. For this purpose, the use of stereo-imaging, computed tomography or any other non planar strain measurement technique seems inevitable.

Another drawback of the proposed procedure lies in the inability to measure strains at the direct vicinity of the cutting tool (i.e. in zones $Z_{I I}$ and $Z_{I I I}$ ). Indeed, experimental constraints are responsible for the lack of data at these locations. Firstly, the small depth of field and the out-of-plane position of the cutting tool lead to a fuzzy delimitation of the tool edges (fuzzy parts of the image around the theoretical position of the cutting tool). Secondly, the nonalignment of the lighting with the optical axis leads to over-exposure of the disoriented facets, especially during the out-of-plane deformation of the segment (white regions of the chip). A correction of these experimental limitations should be addressed in future works.

Finally, achieving a complete comparability between machining experiments and models will require the investigation of thermal effects. Several attempts has been made [1, 16, 2], but the fulfillment of an energy balance over the formation of one segment remains unexplored and surely constitutes the most challenging perspective of this work. 


\section{References}

[1] N.A. Abukhshim, P.T. Mativenga, and M.A. Sheikh. Heat generation and temperature prediction in metal cutting: A review and implications for high speed machining. International Journal of Machine Tools 8 Manufacture, 46:782-800, 2006.

[2] J.P. Arrazola, I. Arriola, and M.A. Davies. Analysis of the influence of tool type, coatings, and machinability on the thermal fields in orthogonal machining of AISI 4140 steels. CIRP Annals - Manufacturing Technology, 58:85-88, 2009.

[3] S. Bahi, M. Nouari, A. Moufki, M. El Mansori, and A. Molinari. A new friction law for sticking and sliding contacts in machining. Tribology International, 44:764$771,2011$.

[4] M. Bornert, F. Brémand, P. Doumalin, M. Dupré, JC. Fazzini, M. Grédiac, F. Hild, S. Mistou, J. Molimard, J-J. Orteu, L. Robert, Y. Surrel, P Vacher, and B. Wattrisse. Assessment of digital image correlation measurement errors: Methodology and results. Experimental Mechanics, 49:353-370, 2008.

[5] T. Braham-Bouchnak. Étude du comportement en sollicitation extrême et de l'usinabilité d'un nouvel alliage de titane aeronautique : le Ti555-3. PhD thesis, École Nationale Supérieure des Arts et Métiers, 2010.

[6] J. Buda. New methods in the study of plastic deformation in the cutting zone. CIRP Annals, 21:17-18, 1972.

[7] M. Calamaz, D. Coupard, and F. Girot. Numerical simulation of titanium alloy dry machining with a strain softening constitutive law. Machining Science and Technology, 14(2):244-257, 2010.

[8] M. Calamaz, J. Limido, M. Nouari, C. Espinosa, D. Coupard, M. Salaün, F. Girot, and R. Chieragatti. Toward a better understanding of tool wear effect through a comparison between experiments and sph numerical modelling of machining hard materials. Int. Journal of Refractory Metals and Hard Materials, 27:595-604, 2009.

[9] M.M. Chaudhri. Subsurface deformation patterns around indentation in workhardened mild steel. Phil. Mag. Lett., 67 67:107-115, 1993.

[10] T.H.C. Childs. A new visio-plasticity technique and a study of curly chip formation. International Journal of Mechanical Sciences, 13:375-387, 1971.

[11] T.H.C. Childs. Friction modelling in metal cutting. Wear, 260:310-318, 2006.

[12] A. Chrysochoos and H. Louche. An infrared image processing to analyse the calorific effects accompanying strain localisation. International Journal of Engineering Science, 38:1759-1788, 2000.

[13] M.G. Cockroft and D.J. Latham. Ductility and workability of metals. J. Inst. Met., 96:33-39, 1968.
[14] C. Courbon. Vers une modélisation physique de la coupe des aciers spéciaux : intégration du comportement métallurgique et des phénomènes tribologiques et thermiques aux interfaces. PhD thesis, Ecole Centrale Université de Lyon, 2011.

[15] H. Ernst and M.E. Merchant. Chip formation, friction and high quality machined surfaces. Surface Treatment of Metals, 29:299-378, 1941.

[16] L. Filice, D. Umbrello, S. Beccari, and F. Micari. On the FE codes capability for tool temperature calculation in machining processes. Journal of Materials Processing Technology, 174:286-292, 2006.

[17] A. Gentel and H.W. Hoffmeiste. Chip formation in machining Ti6Al4V at extremely high cutting speeds. CIRP Annals - Manufacturing Technology, 50(1):49-52, 2001.

[18] G. Germain, F. Morel, J.L. Lebrun, and A. Morel. Machinability and surface integrity for a bearing steel and a titanium alloy in laser assisted machining (optimisation on LAM on two materials). Lasers in Engineering, 17(5-6):329-344, 2007.

[19] E.P. Gnanamanickam, S. Lee, J.P. Sullivan, and S. Chandrasekar. Direct measurement of large-strain deformation fields by particle tracking. Measurement science and technology, 20:095710 (12p), 2009.

[20] Y. Guo, M. Efe, W. Moscoso, D. Sagapuram, K.P. Trumble, and S. Chandrasekar. Deformation field in largestrain extrusion machining and implications for deformation processing. Scripta Materialia, 66:235-238, 2012.

[21] A. Hijazi and V. Madhavan. A novel ultra-high speed camera for digital image processing applications. Measurement Science and Technology, 19:085503 (11p), 2008.

[22] A. Hor, F. Morel, J.-L. Lebrun, and G. Germain. An experimental investigation of the behaviour of steels over large temperature and strain rate ranges. International Journal of Mechanical Sciences, 67:108-122, 2013.

[23] J. Hua and R. Shivpuri. Prediction of chip morphology and segmentation during the machining of titanium alloys. Journal of Materials Processing Technology, 150(12):124-133, 2004.

[24] S.P.F. Jaspers and J. Dautzenberg. Material behaviour in metal cutting: Strains, strain rates and temperatures in chip formation. Journal of Materials Processing Technology, 121:123-135, 2002.

[25] R. Komanduri and R.H. Brown. On the mechanics of chip segmentation in machining. Journal of engineering for industry, 103:33-51, 1981.

[26] R. Komanduri and B.F. von Turkovich. New observations on the mechanisms of chip formation when machining titanium alloys. J. Wear, 69(2):179-188, 1981.

[27] G. Le Goic, H. Favrelière, S. Samper, and F. Formosa. Multi scale modal decomposition of primary form, waviness and roughness of surfaces. Scanning, 33(5):332-341, 2011. 
[28] E.H. Lee and B.W. Shaffer. The theory of plasticity applied to a problem of machining. J.Appl.Mech., 18:405413, 1951.

[29] G. List, G.and Sutter, X.F. Bi, A. Molinari, and A. Bouthiche. Strain, strain rate and velocity fields determination at very high cutting speed. Journal of $M a-$ terials Processing Technology, 213:693-699, 2013.

[30] T. Mabrouki and J.-F. Rigal. A contribution to a qualitative understanding of thermo-mechanical effects during chip formation in hard turning. Journal of Materials Processing Technology, 176:214-221, 2006.

[31] K. Nakayama, M. Arai, and T. Kanda. Machining characteristics of hard materials. CIRP Annals - Manufacturing Technology, 37(1):89-92, 1988.

[32] B. Pan, Z. Lu, and H. Xie. Mean intensity gradient: An effective global parameter for quality assessment of the speckle patterns used in digital image correlation. Optics and Lasers in Engineering, 48:469-477, 2010.

[33] B. Pan, H. Xie, Z. Wang, K. Qian, and Z. Wang. Study on subset size selection in digital image correlation for speckle patterns. Optics express, 16:7037-7048, 2008.

[34] B. Pan, L. Yu, and D. Wu. High-accuracy 2d digital image correlation measurements with bilateral telecentric lenses: Error analysis and experimental verification. Expermental Mechanics, 53:1719-1733, 2013.

[35] T. Pottier, H. Louche, S. Samper, H. Favrelière, F. Toussaint, and P. Vacher. A new filtering approach dedicated to heat sources computation from thermal field measurements. In Photomechanics, Montpellier, France, 2012.

[36] T. Pottier, M-P. Moutrille, J-B. Le-Cam, X. Balandraud, and M. Grédiac. Study on the use of motion compensation techniques to determine heat sources. Application to large deformations on cracked rubber specimens. Experimental Mechanics, 49:561-574, 2009.
[37] J. Pujana, P.J. Arrazola, and J.A. Villar. In-process high-speed photography applied to orthogonal turning. Journal of Materials Processing Technology, 202:475485, 2008.

[38] M.C. Shaw, S.O. Dirke, P.A. Smith, N.H. Cook, E.G. Loewen, and C.T. Yang. Machining titanium. In MIT Rep. (4th edn.) - Massachusetts Institute of Technology, 1954.

[39] B. Shi, H. Attia, and N. Tounsi. Identification of material constitutive laws for machining - part i: An analytical model describing the stress, strain, strain rate, and temperature fields in the primary shear zone in orthogonal metal cutting. In Journal of Manufacturing Science and Engineering, Transactions of the ASME 132., 2010.

[40] A.J. Shih. Finite element analysis of the rake angle effects in orthogonal metal cutting. International Journal of Mechanical Sciences, 1:1-17, 1995.

[41] M. A. Sutton, J. H. Yan, Tiwari V., W. H. Schreier, and J. J. Orteu. The effect of out-of-plane motion on $2 \mathrm{~d}$ and 3d digital image correlation measurements. Optics and Lasers in Engineering, 46:746-757, 2008.

[42] K. Triconnet, K. Derrien, F. Hild, and D. Baptiste. Parameter choice for optimized digital image correlation. Optics and Lasers in Engineering, 47:728-737, 2009.

[43] P. Vacher, S. Dumoulin, F. Morestin, and S. MguilTouchal. Bidimensional strain measurement using digital images. Proc. Inst. Mech. Eng., 213:811-817, 1999.

[44] A. Vyas and M.C. Shaw. Mechanics of saw-tooth chip formation in metal cutting. Journal of manufacturing science and engineering, 121(2):163-172, 1999.

[45] W. Wang, J.E. Mottershead, C.M. Sebastian, and E.A. Patterson. Shape features and finite element model updating from full-field strain data. International Journal of Solids and Structures, 48:1644-1657, 2011. 